\title{
Characterization of lymphocyte responses to peanuts in normal children, peanut-allergic children, and allergic children who acquired tolerance to peanuts
}

\author{
Victor Turcanu, ${ }^{1}$ Soheila J. Maleki, ${ }^{2}$ and Gideon Lack $^{1}$ \\ ${ }^{1}$ Department of Paediatrics, Imperial College Faculty of Medicine, London, United Kingdom \\ ${ }^{2}$ United States Department of Agriculture - Agricultural Research Service - Southern Region Research Center, \\ New Orleans, Louisiana, USA
}

\begin{abstract}
Comparing lymphocyte responses to allergenic and nonallergenic foods could reveal the differences between pathogenic and normal immune responses to foods. Defining the cytokine-producing phenotypes of peanut-specific lymphocytes from peanut-allergic children, children who outgrew peanut allergy, and children who have always tolerated peanuts may be useful for understanding the mechanisms of food tolerance. Investigating immune responses against foods is hindered, however, by the fact that circulating food antigen-specific lymphocytes are very rare. In a novel approach we used carboxyfluorescein succinimidyl ester to detect peanut-specific lymphocytes by flow cytometry. We confirmed that these cells are indeed peanut specific by cloning. Peanut-allergic donors show Th2 polarization of cytokine production by peanut-specific cells (IFN- $\gamma^{\text {low }}$, TNF- $\alpha$ low, IL-4 high, IL-5 high, IL-13 high). Conversely, nonallergic children and children who have outgrown their allergy show Th1 skewing to peanut antigens (IFN- $\gamma^{\text {high }}$, TNF- $\alpha$ high, IL-4 low, IL-5 low, IL-13 low), similarly to nonallergenic food antigens ( $\beta$-lactoglobulin, OVA). This finding suggests that peanut antigens do not intrinsically induce Th2 skewing, but that the type of response depends upon the donor's allergic status. In conclusion, food allergic status is characterized by a Th2 response whereas Th1-skewed responses underlie oral tolerance.
\end{abstract}

J. Clin. Invest. 111:1065-1072 (2003). doi:10.1172/JCI200316142.

\section{Introduction}

Food allergies are common, affecting $6-8 \%$ of infants in their first 3 years of life, but often resolve spontaneously, leading to a decreased prevalence in older children and in adults (1). An exception is peanut allergy: even though it affects only $0.6 \%$ of British 4-year-olds (2), it is the main cause of food-induced anaphylactic shock (3), and it is rarely outgrown. It is not clear whether peanut antigens intrinsically induce a Th2-biased immune response due to their physicochemical properties, or, alternatively, the antigen-specific immune deviation depends on the host immune response. Therefore, comparing $\mathrm{T}$ cell responses to peanuts in peanut allergic (PA) and nonallergic (NA) children, as well as comparing their different $\mathrm{T}$ cell responses to nonallergenic food antigens, might provide useful information.

Received for publication June 10, 2002, and accepted in revised form January 7, 2003

Address correspondence to: Gideon Lack, St. Mary's Hospital, Salton House, Praed Street, London W2 1NY, United Kingdom. Phone: 44-207-886-6384; Fax: 44-207-886-1129;

E-mail: gideon.lack@st-marys.nhs.uk.

Conflict of interest: The authors have declared that no conflict of interest exists.

Nonstandard abbreviations used: peanut allergic (PA); nonallergic (NA); outgrown (OG); egg-allergic (EA); HBSS containing 20 mM HEPES (HBSS+HEPES); tetanus toxoid (TT); phycoerythrin $(\mathrm{PE})$.
Limited and sometimes contradictory information is available concerning the difference in cytokine profiles of peanut-specific lymphocytes from PA and NA donors, possibly due to methodological difficulties: peanut-specific lymphocytes are rare in individuals who tolerate peanuts in their diet also, and there is no data concerning the functional response to peanut antigens of PBMC-derived T cells obtained from the donors that have outgrown their peanut allergy. Such data would provide indications about the mechanism of establishing tolerance to foods and could represent an immunological end point to be achieved by immunotherapy protocols.

Cell surface markers and cytokine-producing phenotype of peanut-specific $T$ cells from PA donors have been studied in the past using peanut-specific clones or cell lines (4-7). In these systems it has been shown that the majority of the clones from PA donors have a Th2 profile (low IFN- $\gamma$ and high IL-4 secretion, as measured by ELISA). Similar results were found when the levels of IFN- $\gamma$ and IL- 4 mRNA were determined in the peanut-activated PBMC cultures $(6,7)$.

The use of peanut-specific clones or cell lines in these studies was justified by the fact that circulating allergen-specific $\mathrm{T}$ cells are present at a very low frequency among PBMCs $(8,9)$. Nevertheless, cloning (or establishing cells lines) typically involves several cycles of Th stimulation in the presence of antigen, feeder cells, and 
exogenous cytokines $(10,11)$. Since this is time and labor consuming, only a limited number of clones can be studied, and the characteristics of the derived antigen-specific cells are altered with regard to antigen responsiveness (12) and cytokine-production phenotype (13). Thus, when stimulated repeatedly in vitro in neutral conditions, human $T$ cells tend to develop by default a Th2-skewed cytokine-producing phenotype $(14,15)$. Moreover, obtaining cell lines or clones against nonallergenic food antigens is relatively difficult because of the very low level of $\mathrm{T}$ cell proliferation induced by such proteins (16). These difficulties explain why the critical comparison between peanutspecific responses and responses to other control food antigens in PA patients has never been made.

We aimed to avoid such problems by establishing a procedure that could allow the identification of food antigen-specific $T$ cells after only one short cycle of in vitro cellular expansion without additional exogenous cytokines and feeder cells. In this respect, we took advantage of the fact that only the antigen-specific Th cells among PBMCs proliferate when cultured in the presence of the respective antigen in stringent culture conditions. To identify these rare antigen (allergen)specific cells that have divided, we labeled the PBMCs with CFSE before setting them in culture.

CFSE is a membrane-permeant dye that binds the amino groups of cytoplasmic proteins with its succinimidyl-reactive group $(17,18)$. When cells divide, CFSElabeled proteins are equally distributed between the daughter cells, thus halving cell fluorescence upon each division. So, in antigen-stimulated cultures, cells are characterized either by high levels of CFSE fluorescence (CFSE ${ }^{\text {high }}$ ), representing the cells that have not divided, or by lower levels of fluorescence (CFSE ${ }^{\text {low }}$ ), the cells that underwent one or more divisions (19-21).

We applied this method to obtain an enriched population of allergen-specific $T$ cells where the allergen-specific cells (CFSE ${ }^{\text {low }}$ ) would be distinguishable from the other cells in culture (that remain highly fluorescent) and further confirmed their antigenic specificity by cloning. This experimental approach allowed us to investigate the cell surface markers and cytokine-secretion phenotype of large numbers of peanut-specific cells. Moreover, using CFSE labeling we could detect low-level immune responses directed against nonallergenic food antigens and thus compare such responses to those observed against peanut antigens in PA children. The cytokine-producing phenotypes of these antigen-specific $T$ cells were then compared with those from children who were never allergic to peanuts, milk, or eggs, as well as children who had recently outgrown their peanut allergy.

\section{Methods}

Patients. Thirteen PA children (average age: 9.42 years; range: 5-16 years; median 9 years), 13 NA children (average age: 9.5 years; range: $6-15$ years; median 9 years), and four "outgrown" (OG) children (average age:
7 years; range: $5-8$ years; median 7 years) were included in this study, which was approved by the Local Research Ethics Committee. The diagnosis of peanut allergy was based on characteristic history of immediate hypersensitivity and at least one of the following diagnostic criteria: peanut skin prick test wheal greater

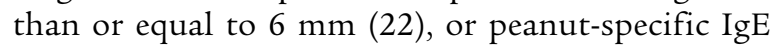
greater than or equal to $15 \mathrm{kU} / \mathrm{l}(23)$, or a positive peanut challenge. The NA children had never reacted to peanut ingestion and were currently consuming peanuts in their diets, tolerating the equivalent of at least one peanut butter sandwich. The four peanut allergy OG children had at least two episodes of immediate hypersensitivity to peanuts between the ages of 1 and 5. Three OGs had their initial diagnosis of peanut allergy confirmed by positive skin prick tests and positive challenges. The fourth patient did not have an initial food challenge to peanuts, but had a typical history and a $12-\mathrm{mm}$ wheal at skin prick test to peanuts. Resolution of peanut allergy was proven in all four children after age 5 by placebo-controlled peanut challenges followed by open challenges. Also, three eggallergic (EA) children were investigated; their allergy was diagnosed on a history of immediate hypersensitivity and at least one of the following criteria: positive skin prick test wheal greater than or equal to $6 \mathrm{~mm}$; egg white-specific IgE greater than or equal to $6 \mathrm{kU} / \mathrm{l}(22)$, or a positive egg challenge. A further five NA children were recruited as their controls.

Reagents. Peanut defatted extract was kindly provided by Henning Løwenstein (ALK Abelló, Horsholm, Denmark). The lyophilized protein was dissolved in PBS, sterilized by filtration, and added to the cell culture at a final concentration of $100 \mu \mathrm{g} / \mathrm{ml}$, while $\beta$-lactoglobulin and OVA (Sigma-Aldrich, Poole, United Kingdom) were used at $200 \mu \mathrm{g} / \mathrm{ml}$. These optimal antigen concentrations were determined by titration in a preliminary experiment. The peanut antigen Ara h2 was purified from peanuts as described (24) and used at a final concentration of $20 \mu \mathrm{g} / \mathrm{ml}$. Tuberculin (PPD; Evans Medical Ltd., Leatherhead, United Kingdom) was dialyzed against PBS and added at $50 \mu \mathrm{g} / \mathrm{ml}$ in culture, while tetanus toxoid (TT; Staatens Serum Institute, Copenhagen, Denmark) was used at $10 \mu \mathrm{g} / \mathrm{ml}$. Fluorochromelabeled Ab's specific for human cytokines (IFN- $\gamma$, IL-4, IL-13, and TNF- $\alpha$ ) were from BD PharMingen (Cowley, United Kingdom). CFSE was from Molecular Probes Inc. (Eugene, Oregon, USA).

PBMC isolation, CFSE labeling, and cell culture. PBMCs were isolated from venous blood by density-gradient separation using Histopaque-1077, then resuspended at $5 \times 10^{7}$ cells $/ \mathrm{ml}$ in HBSS containing $20 \mathrm{mM}$ HEPES (HBSS+HEPES). Immediately before staining, an aliquot of CFSE (5 mM in DMSO) was thawed, diluted 1:10 with HBSS+HEPES, and added to the cell suspension (labeling concentration was $5 \mu \mathrm{M}$ ). PBMCs were incubated with CFSE for 10 minutes at $37^{\circ} \mathrm{C}$ with gentle shaking, then $50 \mathrm{ml} \mathrm{HBSS}+\mathrm{HEPES}$ containing $4 \%$ autologous plasma was added, and the dye excess was 


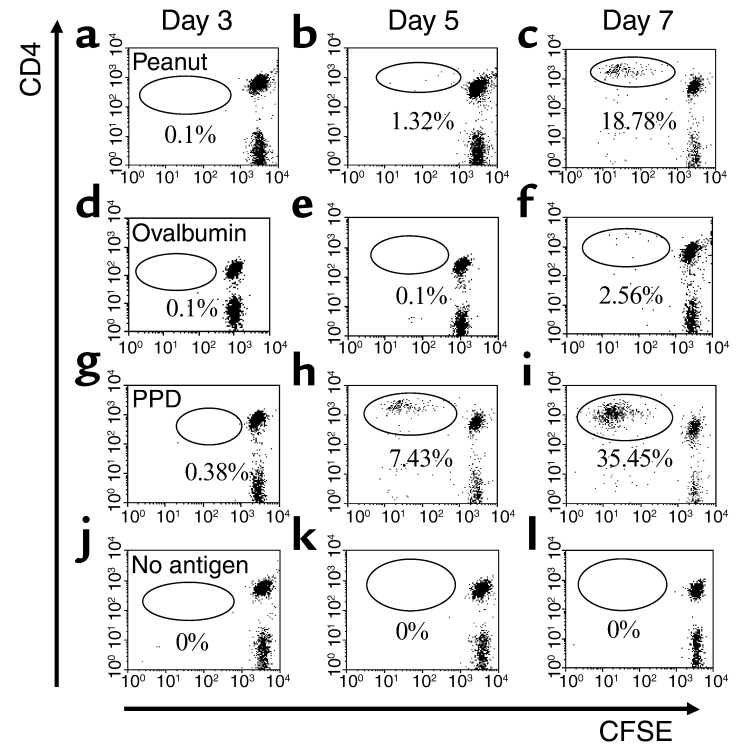

Figure 1

PBMC proliferation monitored using CFSE labeling. PBMCs from a PA donor were isolated, labeled with CFSE, and cultured in the presence of $100 \mu \mathrm{g} / \mathrm{ml}$ peanut extract (a-c), $200 \mu \mathrm{g} / \mathrm{ml}$ OVA (d-f), 50 $\mu \mathrm{g} / \mathrm{ml}$ PPD ( $\mathbf{g}-\mathbf{i})$, or without exogenous antigens $(\mathbf{j}-\mathbf{I})$. On days 3,5 , and 7 after setting the cultures, cells were collected, stained with antiCD4 PE, and analyzed by FACS. Figures represent the percentage of $\left(\mathrm{CFSE}^{\text {low }} \mathrm{CD}^{+}\right)$cells included in the respective gates. Similar results were obtained in three other donors.

washed. CFSE-labeled PBMCs were then cultured with or without antigens in RPMI-1640 containing antibiotics and supplemented with $5 \%$ autologous plasma. Cells were distributed in 24-well flat-bottom plates, at a concentration of $3 \times 10^{6} / 2 \mathrm{ml}$ medium per well.

Cloning allergen-specific lymphocytes. CFSE-labeled PBMCs were cultured with peanut and control antigens for 7 days; then cells were collected and stained with anti-CD4 phycoerythrin (PE). CFSE ${ }^{\text {low }} \mathrm{CD} 4{ }^{+}$cells or $\mathrm{CFSE}^{\text {high }} \mathrm{CD}^{+}$cells were then sorted using a FACSCalibur and distributed (0.3 cells/well) in 96-well U-bottom cell culture plates. Irradiated ( $30 \mathrm{~Gy}$ ) autologous PBMCs $\left(10^{5}\right)$ were added to each well as antigenpresenting cells. To find whether the sorted cells were peanut specific, the lymphocytes separated from among the peanut-stimulated PBMCs were further cultured either with peanut antigens or with OVA (control antigen) or with phytohemagglutinin $(10 \mu \mathrm{g} / \mathrm{ml}$ as a mitogen, to prove the cells' ability to proliferate). To enhance cloning efficiency, in a separate experiment we plated the sorted cells at a concentration of one cell per well and added $10 \mathrm{U} / \mathrm{ml} \mathrm{IL-2}$ in some of the wells. Cloning efficiency was assessed by measuring cell proliferation after 7 more days.

Cell activation and staining for detection of cytokine production by flow cytometry. After 7 days of culture in the presence of antigens, PBMCs were restimulated with PMA $(50 \mathrm{ng} / \mathrm{ml})$ and ionomycin $(1 \mu \mathrm{g} / \mathrm{ml})$ in the presence of brefeldin A $(10 \mu \mathrm{g} / \mathrm{ml})$ for $6 \mathrm{~h}(25)$. The cells were then fixed $(20$ minutes, $2 \%$ paraformalde-
hyde/PBS, $\mathrm{pH} 7.4)$, permeabilized ( $0.2 \%$ saponin), and stained with cytokine-specific Ab's. PBMCs cultured in the presence of the same antigens, but not restimulated with PMA and ionomycin were used as negative controls. At least 10,000 events were collected for each experimental condition, and data were analyzed using the WinMDI 2.8 software (Scripps Research Institute, http://facs.scripps.edu/software.html).

\section{Results}

Antigen stimulation results in the appearance of a population of CFSE low $\mathrm{CD}^{+}$T lymphocytes. PBMCs from PA donors not allergic to egg or to cow's milk were labeled with CFSE and then cultured in the presence of peanut antigens or other control antigens (Figure 1). We found that in the peanut antigen-treated cultures a population of CFSE ${ }^{\text {low }} \mathrm{CD}^{+} \mathrm{T}$ lymphocytes emerges by day 5 , which represents $18.78 \%$ of all the cells in culture by day 7 (Figure 1, a-c). A much smaller population of CFSE $^{\text {low }} \mathrm{CD}^{+} \mathrm{T}$ cells $(2.56 \%)$ emerges by day 7 in the OVA-treated culture (Figure 1, d-f); similar values were seen for the $\beta$-lactoglobulin-treated cultures (data not shown). Comparatively, the recall antigens PPD (Figure $1, \mathrm{~g}-\mathrm{i}$ ) and TT (not shown) induced the emergence of this $\mathrm{CFSE}^{\text {low }} \mathrm{CD}^{+}$earlier and reached a higher proportion of the cells in culture $(35.45 \%$ by day 7 for the PPD-treated PBMCs). Comparatively, when PBMCs are cultured without exogenous antigens (cultures supplemented with $5 \%$ autologous plasma rather than bovine serum to keep background proliferation low), there are practically no emerging CFSE ${ }^{\text {low }}$ cells (Figure $1, j-1$ ), suggesting that the CFSE ${ }^{\text {low }}$ population emerges due to antigen-dependent stimulation. The size of the CFSE ${ }^{\text {low }}$ $\mathrm{CD}^{+} \mathrm{T}$ lymphocyte population significantly correlates with cell proliferation as measured by $\left[{ }^{3} \mathrm{H}\right]$-thymidine incorporation $(r=0.636319 ; P<0.001)$. CFSE-labeling

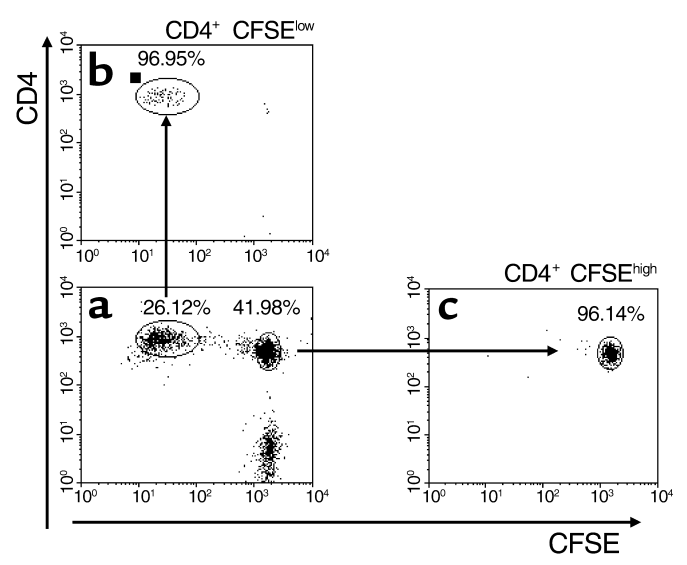

\section{Figure 2}

Flow-cytometry sorting of CFSE ${ }^{\text {low }}$ and CFSE high CD4 ${ }^{+}$lymphocytes. PBMCs from a PA donor were labeled with CFSE and cultured with $100 \mu \mathrm{g} / \mathrm{ml}$ peanut extract for 7 days. Cells were then collected, stained with anti-CD4 PE, and sorted by flow cytometry. Figures show the percentage of the cell populations within the sorting gates from dot plots (a) before sorting, (b) $\mathrm{CD} 4^{+}$CFSElow after sorting, and (c) CD4+ CFSEhigh after sorting. 


\section{Table 1}

Cloning efficiency after sorting for CFSE ${ }^{\text {low }}$ and CFSEhigh Th lymphocytes from PBMCs cultured in the presence of peanut antigens.

Without IL-2 (\%) With IL-2 (\%)

$\begin{array}{lcc}\text { CFSElow } & & \\ \text { Without antigens } & 0 & 0 \\ \text { Peanut antigens } & 33 & 91.6 \\ \text { OVA (control antigen) } & 0 & 0 \\ \text { Phytohemagglutinin } & 58.3 & 100 \\ \text { CFSEhigh cells } & & \\ \text { Without antigens } & 0 & 0 \\ \text { Peanut antigens } & 0 & 0 \\ \text { OVA (control antigen) } & 0 & 83.3 \\ \text { Phytohemagglutinin } & 58.3 & \end{array}$

PBMCs from a PA donor were CFSE-labeled and cultured in the presence of peanut antigens for 7 days. The cells were then collected, stained with antiCD4, and the CFSElow and the CFSEhigh Th cells were sorted by flow cytometry, then subsequently cloned by culture in the presence of different antigens and feeder cells. Figures represent the percentage of wells showing cell proliferation (as a measure of cloning efficiency).

of PBMCs does not interfere with antigen-specific proliferation (data not shown).

Cloning of $\mathrm{CFSE}^{\text {low }} \mathrm{CD}^{+} \mathrm{T}$ cells sorted from CFSElabeled PBMCs cultured in the presence of peanut antigens demonstrates that they are a peanut-specific population. Even though the CFSElow population from peanut-treated PBMC cultures correlates with cell proliferation, this does not prove its antigen specificity. This CFSElow population could have resulted in response to a nonspecific mitogenic effect or as a generalized bystander $\mathrm{T}$ cell response. Therefore, we confirmed the antigen specificity of these cells in a series of cloning experiments. PBMCs from a PA donor were labeled with CFSE and cultured in the presence of peanut antigens for 7 days. The cells were then collected, stained with anti-CD4 PE (Figure 2a), and sorted using a FACSCalibur gating on the CFSElow (Figure 2b) and, respectively, CFSE high populations (Figure 2c). Dead cells were gated out using 7-aminoactinomycin $\mathrm{D}$ (not shown). Both CFSE ${ }^{\text {low }}$ and CFSE ${ }^{\text {high }}$ cells were more than $96 \%$ pure, as shown by postsorting reacquisition. After sorting, the cells were recovered, diluted in culture medium with or without IL-2, and distributed together with irradiated feeder cells and antigens in 96-well U-bottom plates. Cloning efficiency was assessed after 7 days by measuring proliferation using $\left[{ }^{3} \mathrm{H}\right]$-methyl-thymidine incorporation (Table 1 ). We thus found that $\mathrm{CFSE}^{\text {low }}$ cells emerging in the presence of peanut antigens are predominantly peanut specific: they proliferate in the presence of peanut antigens (91.6\% in the presence of IL-2) but do not respond to other antigens (OVA). Conversely, CFSE high lymphocytes did not proliferate in the presence of peanut antigens, even though they were able to proliferate (as shown when activated by the mitogen phytohemagglutinin). Similarly, in a separate experiment (data not shown) PPD-specific T cells were cloned from a sorted $\mathrm{CFSE}^{\text {low }}$ population that had emerged following PBMC stimulation with PPD. These results clearly demonstrate that $\mathrm{CFSE}^{\text {low }}$ cells are antigen specific.

Peanut-specific $\mathrm{T}$ cells display marked $\mathrm{Th} 2$ polarization in PA children, contrasting with the Th1-bias of responses induced by nonallergenic food proteins. We show the responses to peanut antigens, to control nonallergenic food antigens ( $\beta$-lactoglobulin, OVA), and to recall antigens (PPD and TT) in a typical PA donor who was not allergic to eggs and milk (Figure 3 ). We express the results as the percentage of cytokinepositive cells among the CFSE ${ }^{\text {low }}$ (antigen-specific) lymphocytes, counting at least 10,000 cells per sample. Th1-polarization is characteristic for the response to PPD (a typical Th1 antigen; ref. 26); as seen in Figure 3a $(95.86 \%$ of PPD-specific T cells secreted IFN- $\gamma$, while only $0.22 \%$ produced IL- 4 ). When PBMCs were stimulated with TT, a response involving the production of Th2 2 cytokines emerged (as seen in Figure 3b, 72.39\% of TT-specific T cells secreted IFN- $\gamma$, while $9.41 \%$ produced IL-4). We then assessed the responses to the nonallergenic food proteins $\beta$-lactoglobulin (Figure $3 c$ ) and OVA (Figure 3d) and found a clear Th1-skewing $(92.44 \%$ and $93.68 \%$, respectively, of antigen-specific lymphocytes produce IFN- $\gamma$, while less than $1 \%$ IL-4-secreting cells could be detected). In comparison, when PBMCs from the PA donor were cultured in the presence of peanut antigens, the response of the peanut-specific lymphocytes was Th2 skewed (Figure 3e: $47.65 \%$ IFN- $\gamma$ - and $10.84 \%$ IL-4-secreting cells).

We investigated the production of other cytokines (TNF- $\alpha$ that is produced by Th0 and Th 1 cell populations; IL-13 that is produced by Th0 and Th2 cells) and also IL-5 (data not shown) in response to the same antigens as mentioned above (Figure 4). We compared the Th1/Th2 cytokine-producing antigen-specific lym-

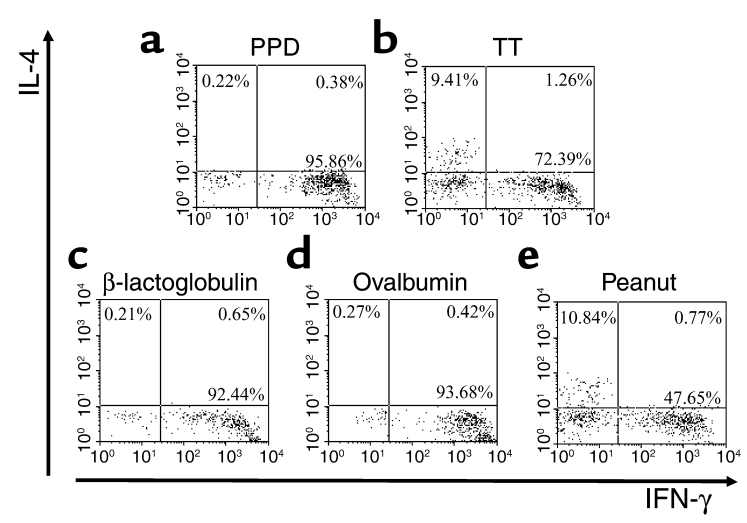

Figure 3

Th2 skewing of peanut-specific responses from a PA donor contrasts with the Th1-biased response induced by nonallergenic food antigens. PBMCs isolated from a PA donor, labeled with CFSE, and cultured in the presence of antigens for 7 days were restimulated with PMA and ionomycin in the presence of brefeldin A for $6 \mathrm{~h}$, then cytokine production was assessed using intracellular cytokine staining. Plots show IFN- $\gamma / \mathrm{IL}-4$ production (percentage of cytokine positive cells) by the antigen-specific (CFSElow) cells specific for PPD (a), $\Pi \mathrm{T}(\mathbf{b}), \beta$-lactoglobulin (c), OVA (d), and peanuts (e). 

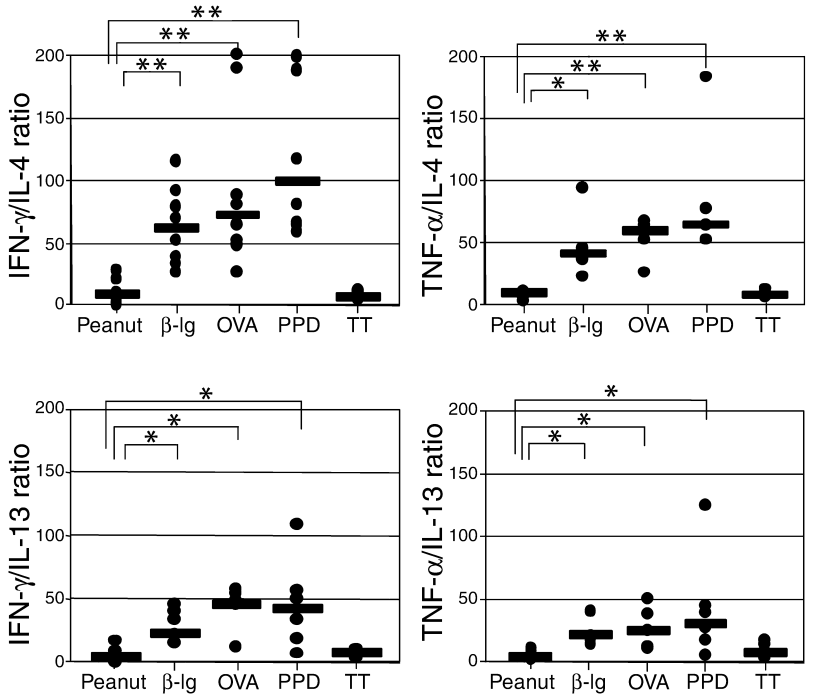

Figure 4

Th subset polarization of antigen-specific T lymphocytes from PA donors $(n=9)$. PBMCs were isolated, CFSE labeled, and cultured in the presence of antigens for 7-8 days, then the cytokine-producing phenotype of antigen-specific (CFSElow) cells was assessed by flow cytometry. Th subset skewing was determined as ratios of CFSElow cell percentages that produce Th1-type (IFN- $\gamma$ ) or Th2-type (IL-4) cytokines (and also TNF- $\alpha$ or IL-13). Points represent data from different individuals, while bars show median values. Significance was determined using the paired $t$ test for log-transformed data $\left({ }^{*} P<0.05 ;{ }^{*} P<0.02\right)$. $\beta$-lg, $\beta$-lactoglobulin.

phocytes ratios for all antigens studied in nine PA donors. We found a highly significant difference between the Th2-skewed peanut-specific response and that induced by $\beta$-lactoglobulin, OVA, and PPD. On the other hand, there was no significant difference between the peanut-response and that induced by TT, confirming their Th2-bias. Similar differences were observed when considering other cytokine ratios measured, corroborating a Th2 skewing (fewer IFN- $\gamma$ and TNF- $\alpha$ and more IL-4, IL-5, and IL-13-producing cells) in peanut-specific $\mathrm{T}$ cells.

Peanut-specific T cells display marked Th1 skewing in children who do not have peanut allergy. Whereas peanut-specific lymphocytes derived from PA children PBMCs displayed a Th2-biased cytokine-producing phenotype, this could be explained by intrinsic proallergenic properties of the peanut proteins rather than by the allergic status of the donors. Therefore, we compared the Th profiles for these food antigens in children who were not allergic to peanuts, milk, or eggs $(n=9)$. We found that in these children the peanut-specific response is Th1 skewed, as is the case with the other control food antigens, with no significant difference between the cytokine profiles induced by the three foods (Figure 5). We did find, however, significant differences $(P<0.02)$ between the responses to peanuts and to the typical Th2 antigen, TT, for all cytokines investigated. Therefore, the differences between peanut-specific Th responses in PA, as well as in NA children, reflect the allergic status of the donors.
Peanut-specific T cells from children who have recently outgrown their peanut allergy also display Th1 skewing. More recently, it has become apparent that clinical resolution may occur in a subgroup of children with peanut allergy (OGs). We studied the peanut-specific $\mathrm{T}$ cell response in four OG children who have suffered from peanut allergy but who are now tolerating peanuts in their diet. We found that the peanut-specific cytokine profiles in these children are similar to those in children who have no history of peanut allergy. Statistically, there was a clear difference between cytokine phenotypes from PA children and those from NA or OG donors, but no significant difference between NA and OG children (Figure 6). Therefore, acquisition of tolerance in children previously allergic to peanuts is accompanied by a shift in the cytokine phenotype of peanutspecific lymphocytes from a Th2 to a Th1 profile.

Similar differences between PA and NA donors can be observed in responses to purified Ara b2 peanut antigen. Crude defatted peanut extract contains numerous antigens that might induce diverse responses, and lectins could potentially have nonspecific stimulatory effects. We thus investigated the immune response directed to purified Ara h2 using PBMCs isolated from PA and NA donors. We observed a similar pattern of skewing in PA donors (Figure 7).

OVA-specific $\mathrm{T}$ cells display marked Th2 polarization in EA children, contrasting with the Th1 bias of the response in NA children. To determine whether
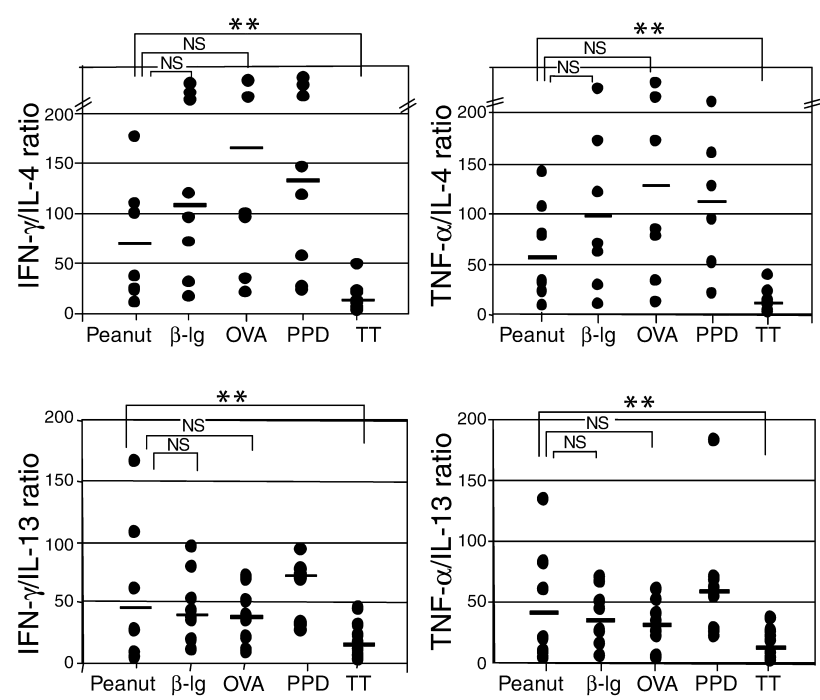

Figure 5

Th subset polarization of antigen-specific T lymphocytes from NA donors $(n=9)$. PBMCs were isolated, CFSE labeled, and cultured in the presence of antigens for 7 days, then the cytokine-producing phenotype of antigen-specific (CFSElow) cells was assessed by flow cytometry. Th subset skewing was determined as ratios of CFSElow cell percentages that produce Th1-type (IFN- $\gamma$ ) or Th2-type (IL-4) cytokines (and also TNF- $\alpha$ and IL-13). Points represent data from different individuals, while bars show median values. Significance was determined using the paired $t$ test for log-transformed data $\left({ }^{*} P<0.05 ;{ }^{*} P<0.020\right)$. 

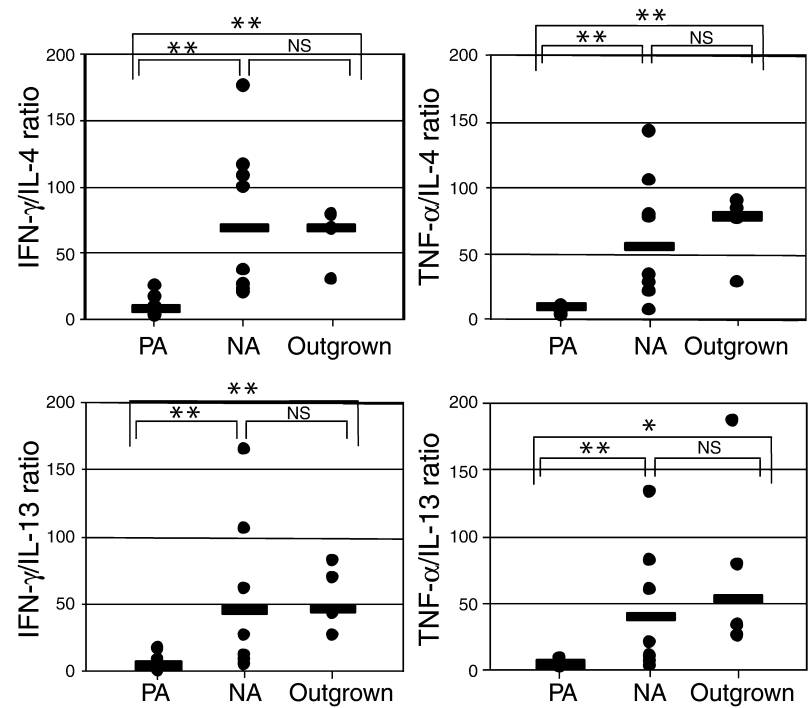

Figure 6

Comparison of the Th subset polarization of antigen-specific T lymphocytes from PA $(n=9)$, NA $(n=9)$, and PA-outgrown donors $(n=4)$. PBMCs were isolated, CFSE labeled, and cultured in the presence of antigens for 7 days, then the cytokine-producing phenotype of antigen-specific (CFSElow) cells was assessed by flow cytometry. Th subset skewing was determined as ratios of CFSElow cell percentages that produce Th1-type (IFN- $\gamma$ ) or Th2type (IL-4) cytokines (and also TNF- $\alpha$ and IL-13). Points represent data from different individuals, while bars show median values. Significance was determined using the Mann-Whitney test $\left({ }^{*} P<0.05 ;{ }^{*} P<0.02\right)$.

the differences in cytokine responses that we observed between the case of PA and NA children is specific for peanut allergy, we studied OVA-specific responses in three EA children and compared them with five NA donors. We found similar TH2 skewing in the OVA-specific $T$ cells of egg allergic compared with tolerant donors: EA donors have fewer IFN- $\gamma-$ secreting cells $(47.5 \% \pm 5.3 \%)$ compared with NA donors $(77.6 \% \pm 5.6 \%, P=0.01)$, fewer TNF- $\alpha$-secreting cells $(42.2 \% \pm 1.7 \%$ versus $68.9 \% \pm 6.9 \%$ for the NA, $P=0.01)$ but more IL-4 secretors $(3.9 \% \pm 1.1 \%$ versus $0.9 \% \pm 0.2 \%, P=0.01$ ), and more IL-13-secreting cells $(5.5 \% \pm 2.8 \%$ versus $1.3 \% \pm 0.4 \%, P=0.05)$.

\section{Discussion}

Previous studies on cytokine responses to food allergens have never compared allergen-specific T lymphocytes from food-allergic donors with $\mathrm{T}$ lymphocytes that respond to nonallergenic foods, largely due to technical difficulties arising from the low frequency of such cells in the blood. We have overcome this difficulty using a novel application of CFSE-labeling. We demonstrated by cloning that this new method allowed the identification of antigen-specific cells after shortterm in vitro antigen-stimulation of PBMCs, even when the expanded antigen-specific cells represent a small percentage of all the cells in the culture. Since CFSElabeling is relatively straightforward, does not interfere with lymphocyte proliferation, and requires only small numbers of cells, this technique could be used to study other immune disorders such as type I diabetes mellitus and multiple sclerosis, which are characterized by abnormal antigen-specific $\mathrm{T}$ cell responses.

In the present work, we used CFSE labeling to investigate food antigen-specific $\mathrm{T}$ cell responses in PA children, in NA children, and in OG children. We found that the control nonallergenic foods ( $\beta$-lactoglobulin, OVA) induce a Th1-skewed cytokine profile regardless of the allergic status (PA, NA, or OG) of the donors. This Th1-skewing induced by nonallergenic food antigens is characterized by a high production of IFN- $\gamma$ and TNF- $\alpha$, almost undetectable production of IL- 4 and IL-5, and relatively low production of IL-13. This phenotype was similar to that induced in the same donors by PPD, a typical Th1 antigen, and suggests that oral tolerance to food antigens is characterized by a Th1skewed immune response.

In agreement with previous studies of peanut-specific cell clones derived from PA donors (4-7), we found that peanut-specific responses are Th2 skewed in PA children. We have extended these findings to show that these responses to peanuts from PA children are clearly different from the Th1-like responses that we found in NA donors. Furthermore, we have shown, we believe for the first time, that resolution of peanut allergy is accompanied by acquisition of peanut-specific Th1 responses.

The same differences were found between PA and NA donors using purified Ara h2, indicating that
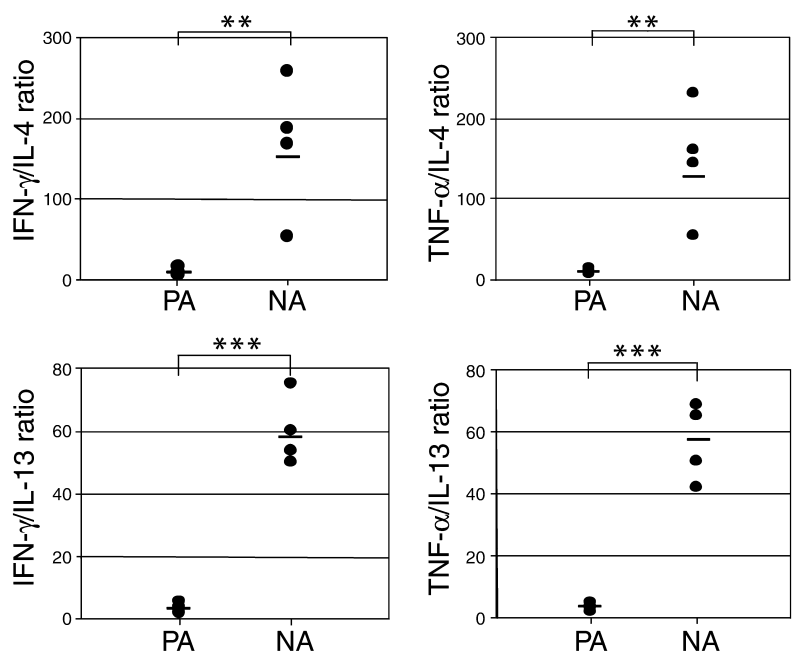

\section{Figure 7}

Comparison of the responses of Ara h2-specific T lymphocytes from PA $(n=4)$ and NA donors $(n=4)$. PBMCs were isolated, CFSE labeled, and cultured in the presence of $20 \mu \mathrm{g} / \mathrm{ml}$ purified Ara h2 for 7 days, then the cytokine-producing phenotype of antigen-specific (CFSElow) cells was assessed by flow cytometry. Th-subset skewing was determined as ratios of CFSElow cell percentages that produce Th1-type (IFN- $\gamma$ ) or Th2-type (IL-4) cytokines (and, respectively, TNF- $\alpha$ and IL-13). Points represent data from different individuals, while bars show median values. Significance was determined using the Mann-Whitney test $\left({ }^{*}{ }^{*} P<0.001 ;{ }^{*} P<0.02\right)$. 
these differences in cytokine skewing occur with single antigen stimulation and do not represent nonspecific responses to lectins.

Furthermore, we were able to show that children with egg allergy and milk allergy (data not shown) similarly display Th2 skewing compared with nonallergic children. These differences in cytokine skewing are therefore not specific to peanuts, but underlie immunological responses in diverse food allergies.

Even though the percentage of IFN- $\gamma$-secreting lymphocytes is much lower than in their NA counterparts, nevertheless $35-50 \%$ of peanut-specific cells produce IFN- $\boldsymbol{\gamma}$. This IFN- $\boldsymbol{\gamma}$-secreting population is separate from the IL-4/IL-5-secreting population and could represent a distinct cell phenotype. This is similar to the response to tetanus, which is regarded as a Th2dominant response (27), but nevertheless has been shown to include a Th1 component (28). Indeed, the existence of multiple lymphocyte subsets among circulating $\mathrm{T}$ cells from EA patients was shown using ovomucoid overlapping peptides (29); however, the dominant IFN- $\gamma$ and TNF- $\alpha$ responses observed in our study of the peanut-specific cells from NA and OG donors suggest that Th1 responses underlie both nonallergic status and resolution of peanut allergy.

Our results agree with several studies concerning immune responses to food antigens. In one of these studies, all the antigen-specific $\mathrm{T}$ cell clones isolated from the peripheral blood of children sensitized to cow's milk or egg were shown to produce high amounts of IFN- $\gamma$ in addition to IL-4, when stimulated with concanavalin A (30).

A recent study attempted to compare the $\beta$-lactoglobulin-specific responses in PBMCs and Peyer's patch-derived lymphocytes from children that were not allergic to cow's milk (31). The authors describe a Th1-polarized (high IFN- $\gamma$ secretion) cytokine response of milk antigen-stimulated lymphocytes isolated from Peyer's patches. However, no comparison could be made with the $\beta$-lactoglobulin-specific response in peripheral blood $T$ cells, presumably due to the low frequency of antigen-specific circulating precursors. Using CFSE labeling, we have overcome this difficulty and compared the immune response to both allergenic and nonallergenic food antigens. This technique brings three significant advantages. First, we avoid repetitive cycles of in vitro stimulation in the presence of exogenous cytokines and feeder cells that likely induce functional bias. Second, the identification of rare antigen-specific $T$ cells by flow cytometry allows us to study large numbers of cells $(10,000$ events or more), conferring superior statistical power in comparison with the analysis of a limited number of lymphocyte clones. Third, detecting cytokine production in large numbers of heterogeneous, non-clonal antigen-specific lymphocytes allows a better investigation of complex, distinct $\mathrm{T}$ cell populations.

Our data argue against the existence of intrinsic Th2skewing properties of food allergens. It has been sug- gested that certain antigens might possess intrinsic properties (physicochemical or biological) that render them allergenic $(32,33)$. In our study, we found that the same peanut antigen extract induces a Th2-skewed response in PA donors and a Th1-skewed response in NA children. Therefore, the pattern of cytokine production seems to depend on the host immune response rather than on inherent properties of the allergen.

In conclusion, we have used a novel approach to identify antigen-specific $T$ cells that may have important applications in studying both allergic and autoimmune disorders. Using this method, we have shown that peanut antigens induce a Th2-biased response in cells derived from the PBMCs of PA children, while nonallergenic food antigens induce a Th1skewed response. The resolution of peanut allergy is accompanied by a shift to a Th1 cytokine profile. Thus, by correlating immune responses to clinical phenotypes, we provide a context against which to evaluate future immunomodulatory treatments that aim to induce oral tolerance to peanuts.

\section{Acknowledgments}

We thank Kevin Babb for technical assistance, Graham Roberts from the Department of Paediatrics, St. Mary's Hospital in London, United Kingdom, for help with statistical analysis, and Henning Løwenstein and Gitte Nordskov-Hansen from ALK Abelló for providing the defatted peanut extract. Gideon Lack is supported by the Aimwell Foundation. This study was funded by the Food Standards Agency, United Kingdom, and the National Peanut Board, USA.

1. Bock, S.A. 1987. Prospective appraisal of complaints of adverse reactions to foods in children during the first 3 years of life. Pediatrics. 79:683-688. 2. Hourihane, J.O’B., Dean T.P., and Warner J.O. 1998. Peanut allergic subjects' peripheral blood mononuclear cell proliferative responses to crude peanut protein. Clin. Exp. Allergy. 28:163-168.

3. Sampson, H.A. 2001. Immunological approaches to the treatment of food allergy. Ped. Allergy Immunol. 12:91-96.

4. de Jong, E.C., et al. 1996. Food allergen (peanut)-specific TH2 clones generated from the peripheral blood of a patient with peanut allergy. J. Allergy Clin. Immunol. 98:73-81.

5. Higgins, J.A., Lamb, J.R., Lake, R.A, and O'Hehir, R.E. 1995. Polyclonal and clonal analysis of human CD4+ T-lymphocyte responses to nut extracts. Immunology. 84:91-97.

6. Laan, M.P., et al. 1998. CD4+ cells proliferate after peanut-extract specific and CD8+ cells proliferate after polyclonal stimulation of PBMC of children with atopic dermatitis. Clin. Exp. Immunol. 28:35-44.

7. Dorion, B.J., et al. 1994. The production of interferon-gamma in response to a major peanut allergen, Ara $b$ II, correlates with serum levels of IgE anti-Ara $b$ II. J. Allergy Clin. Immunol. 93:93-99.

8. Burastero, S.E., Fenoglio, D., Crimi, E., Brusasco, V., and Rossi, G.A. 1993. Frequency of allergen-specific T lymphocytes in blood and bronchial response to allergen in asthma. J. Allergy Clin. Immunol. 91:1075-1081.

9. Neumann, C., Gutgesell, C., Fliegert, F., Bonifer, R., and Herrmann, F. 2001. Comparative analysis of the frequency of house dust mite specific and nonspecific Th1 and Th2 cells in skin lesions and peripheral blood of patients with atopic dermatitis. J. Mol. Med. 74:401-406.

10. Gurka, G., Ohman, J., and Rosenwasser, L.J. 1989. Allergen-specific human $T$ cell clones: derivation, specificity and activation requirements. J. Allergy Clin. Immunol. 83:945-954.

11. Spits, H., and Yssel, H. 1996. Cloning of human T and natural killer cells. Methods. 9:416-421.

12. Paliard, X., de Vries, J., and Spits, H. 1991. Comparison of lymphokine secretion and responsiveness of human T cell clones isolated in IL-4 and IL-2. Cell Immunol. 135:383-393.

13. Cohen, S., Webb, L., and Feldmann, M. 1996. The method of deriving 
human T-cell clones alters the proportion of IL-10-producing cells. Immunology. 87:343-347.

14. Demeure, C.E., et al. 1995. Human naive CD4 T cells produce interleukin- 4 at priming and acquire a Th2 phenotype upon repetitive stimulations in neutral conditions. Eur. J. Immunol. 25:2722-2725.

15. Yang, L.-P., Byun, D.-G., Demeure, C.E., Vezzio, N., and Delespesse, G. 1995. Default development of cloned human naive CD4 T cells into interleukin-4- and interleukin-5-producing effector cells. Eur. J. Immunol. 25:3517-3520

16. Agata, H., et al. 1993. Evaluation of lymphocyte proliferative responses to food antigens with regard to age and food-specific $\mathrm{IgE}$ antibodies in food-sensitive atopic dermatitis. J. Investig. Allergol. Clin. Immunol. 3:174-177.

17. Weston, S.A., and Parish, C.R. 1990. New fluorescent dyes for lymphocyte migration studies: analysis by flow cytometry and fluorescence microscopy. J. Immunol. Methods. 133:87-97.

18. Lyons, A.B., Hasbold, J., and Hodgkin, P.D. 2001. Flow cytometric analysis of cell division history using dilution of carboxyfluorescein diacetate succinimidyl ester, a stably integrated fluorescent probe. Methods Cell Biol. 63:375-398.

19. Lyons, A.B., and Parish, C.R. 1994. Determination of lymphocyte division by flow cytometry. J. Immunol. Methods. 171:131-137.

20. Angulo, R., and Fulcher, D.A. 1998. Measurement of Candida-specific blastogenesis: comparison of carboxyfluorescein succinimidyl ester labeling of $\mathrm{T}$ cells, thymidine incorporation and CD69 expression. Cytometry. 34:143-151.

21. Fulcher, D.A., and Wong, S. 1999. Carboxyfluorescein succinimidyl ester-based proliferative assays for assessment of T cell function in the diagnostic laboratory. Immunol. Cell. Biol. 77:559-564.

22. Hill, D.J., Hosking, C.S., and Reyes-Benitom, L.V. 2001. Reducing the need for food allergen challenges in young children: a comparison of in vitro and in vivo tests. Clin. Exp. Allergy. 31:1031-1035.
23. Sampson, H.A., and Ho, D.G. 1997. Relationship between food-specific $\mathrm{IgE}$ concentrations and the risk of positive food challenges in children and adolescents. J. Allergy Clin. Immunol. 100:444-451.

24. Sen, M., et al. 2002. Protein structure pays a critical role in peanut allergen stability and may determine immunodominant IgE-binding epitopes. J. Immunol. 169:882-887.

25. Prussin, C., and Metcalfe, D.D. 1995. Detection of intracytoplasmic cytokine using flow cytometry and directly conjugated anti-cytokine antibodies. J. Immunol. Methods. 188:117-128.

26. Kamal, A.M., Smith, S.F., De Silva Wijayasinghe, M., Solito, E., and Corrigan, C.J. 2001. An annexin 1 (ANXA1)-derived peptide inhibits prototype antigen-driven human T cell Th1 and Th2 responses in vitro. Clin. Exp. Allergy. 31:1116-1125.

27. Parronchi, P., et al. 1991. Allergen- and bacterial antigen-specific T-cell clones established from atopic donors show a different profile of cytokine production. Proc. Natl. Acad. Sci. U. S. A. 88:4538-4542.

28. Rowe, J., et al. 2000. Antigen-specific responses to diphtheria-tetanusacellular pertussis vaccine in human infants are initially Th2 polarised. Infect. Immun. 68:3873-3877.

29. Reekers, R., et al. 1996. The role of circulating food antigen-specific lymphocytes in food allergic children with atopic dermatitis. Br. J. Dermatol. 135:935-941.

30. Holen, E., Bolann, B., and Elsayed, S. 2001. Novel B and T cell epitopes of chicken ovomucoid (Gal d1) induce T cell secretion of IL-6, IL-13 and IFN-gamma. Clin. Exp. Allergy. 31:952-964.

31. Nagata, S., et al. 2000. Human Peyer's patch T cells are sensitized to dietary antigen and display a Th cell type 1 cytokine profile. J. Immunol. 165:5315-5321.

32. Aalberse, R.C., and Stapel, S.O. 2001. Structure of food allergens in relation to allergenicity. Pediatr. Allergy Immunol. 12:10-14.

33. Huby, R.D.J., Dearman, R.J., and Kimber, I. 2000. Why are some proteins allergens? Toxicol. Sci. 55:235-246. 